\title{
The Addition of the Charlson Comorbidity Index to the GRACE Risk Prediction Index Improves Prediction of Outcomes in Acute Coronary Syndrome
}

\author{
Steven R. Erickson, PharmD, Emily Cole, PharmD,, ${ }^{1,2}$ Eva Kline-Rogers, RN, NP, ${ }^{3}$ and Kim A. Eagle, MD ${ }^{4}$
}

\begin{abstract}
Patients with cardiovascular disease have increased risk of poor outcomes when coexisting illnesses are present. Clinicians, administrators, and health services researchers utilize risk adjustment indices to stratify patients for various outcomes. The GRACE Risk Prediction Index (GRPI) was developed to risk stratify patients who experienced an acute coronary syndrome (ACS) event. GRPI does not account for the presence of comorbid conditions. The objective of this study was to compare the ability of the GRPI and the Charlson Comorbidity Index (CCI), used independently or combined, to predict mortality or secondary coronary events in patients admitted for ACS. Data were obtained from an academic health system's ACS registry. Outcomes included inpatient and 6-month postdischarge mortality and occurrence of secondary cardiovascular events or revascularization procedures. Logistic regression derived C statistics for CCI, GRPI, and CCI-GRPI predictive models for each outcome. Likelihood ratio tests determined the contribution of CCI when added to GRPI models. Complete data were available for 1202 patients. The GRPI model had the greatest $C$ statistic when predicting inpatient mortality (0.73); the GRPI-CCI combined model C statistic was 0.81 when predicting death during the follow-up period; and C statistics for all 3 models were similar in predicting secondary events $(0.57-0.60)$. The likelihood ratio analysis demonstrated that adding CCI to GRPI models was beneficial primarily for predicting secondary events. CCI is a useful addition to GRPI when predicting future cardiac-related events or mortality after an ACS event. It is an acceptable alternative to the GRPI model if data to construct GRPI are not available. (Population Health Management 2014;17:54-59)
\end{abstract}

\section{Introduction and Background}

$\mathbf{O}$ ver 16 million People in the United States have the diagnosis of coronary artery disease. ${ }^{1}$ Coronary artery disease is a subset of conditions of the vascular system. Cardiovascular disease includes all diseases of the circulatory system, including coronary artery disease, stroke, peripheral artery disease, and diseases of the veins. ${ }^{1}$ Acute coronary syndrome (ACS) occurs when a coronary artery becomes acutely occluded, reducing blood flow to dependent myocardium. ACS includes unstable angina and myocardial infarction. Over 935,000 people experience a myocardial infarction every year. ${ }^{1}$ Patients who experience an ACS event frequently have comorbid conditions. For example, of patients aged 65 years of age and older who presented for emergent treatment related to an ACS event, $6.3 \%$ had 1 comorbid condition, $23.1 \%$ had 2 comorbidities, and $68.5 \%$ had 3 or more comorbid conditions. ${ }^{2}$

Multimorbidity, a term used to describe the coexistence of multiple illnesses, is associated with increased mortality; 2 or more chronic diseases result in a 2-fold or more increase in mortality risk. ${ }^{3,4}$ Patients with cardiovascular disease have increased risk of mortality when coexisting diabetes and chronic kidney disease are present. ${ }^{5}$ For patients with an acute myocardial infarction, comorbidity is also significantly associated with inpatient mortality. ${ }^{6,7}$ Finally, a study of over 1400 patients with coronary artery disease who underwent cardiac catheterization and were followed for 10 years demonstrated that comorbid disease is strongly associated with long-term survival. This particular study used the

\footnotetext{
${ }^{1}$ University of Michigan College of Pharmacy, ${ }^{3}$ Michigan Cardiovascular Outcomes Research and Reporting Program (M-CORRP), and ${ }^{4}$ Cardiovascular Center, University of Michigan Health System, Ann Arbor, Michigan.

${ }^{2}$ Department of Pharmacy, University of Pittsburgh, Pittsburgh, Pennsylvania.
} 
Charlson Comorbidity Index (CCI) to quantitate the burden of coexisting illness. ${ }^{8}$

The CCI was developed to assess the 1-year mortality risk in patients with comorbidities, and is widely used. ${ }^{9}$ The index consists of 10 general comorbidity categories: myocardial, vascular, pulmonary, neurologic, endocrine, renal, liver, gastrointestinal, cancer/immune, and miscellaneous. The CCI is further divided into specific comorbidities (eg, peptic ulcer is classified under gastrointestinal). The index is weighted to account for the prevalence and seriousness of the disease; for example, a myocardial infarction is given a score of 1 , whereas AIDS is weighted as a $6 .{ }^{9}$ Charlson et al found that the index directly correlated increasing ranks of comorbidity with greater mortality rates. ${ }^{9}$

The Global Registry of Acute Coronary Events or the GRACE risk prediction index (GRPI) was developed as a tool for use by clinicians to estimate the overall risk of mortality in patients during hospitalization for an ACS event and for the 6-month period after discharge. The index may be used as a decision tool when developing treatment strategies. Risk indices also may be useful for predicting hospital readmission rates, both to identify which patients would benefit most from care transition interventions and to standardize readmission risk rates for the purposes of comparisons of hospital quality.

The GRPI is a condition-specific risk-adjustment index that is able to predict mortality during hospitalization for the index event, as well as at time periods of 6 months to 4 years after the index event. ${ }^{10-14}$ The GRPI includes 9 independent risk factor variables: age, history of myocardial infarction, history of heart failure, increased pulse rate at presentation, lower systolic blood pressure at presentation, elevated initial creatinine level, elevated initial serum cardiac biomarker levels, ST-segment depression on presenting electrocardiogram, and not having a percutaneous coronary intervention performed in the hospital. ${ }^{12}$ The GRPI performed well in all forms of ACS (C statistic of at least 0.70), proving to be a simple, useful model to predict mortality risk and guide the management of patients. ${ }^{12}$

Recent studies have attempted to improve the predictive ability of the GRPI by adding biomarkers or additional clinical measures. ${ }^{15-17}$ These studies demonstrated incremental increases in predictive ability when additional information was added to the index. No studies have attempted to improve the predictive ability of models using the GRPI by combining with an index of comorbidity such as the CCI. It may be reasoned that accounting for the contribution of comorbid conditions that are themselves associated with mortality would improve the predictive ability of modeling of mortality of patients with ACS. The present study utilized an ACS registry to assess the relative contribution of the CCI when added to models that include the GRPI in predicting mortality and secondary events or procedures. The goal of this project was to determine if the addition of the CCI to the GRPI could improve the predictive ability of mortality and secondary events over the GRPI. Three end points were used for this study: inpatient mortality during hospitalization for the index ACS event; outpatient mortality within 6 to 12 months after discharge for the index event; and occurrence of secondary events or procedures during the follow-up period after the index event.

\section{Methods}

\section{Subjects in the Acute Coronary Syndrome Registry}

This study used data obtained from a registry of patients admitted to and discharged from a large university-affiliated medical center for the treatment of ACS. The registry uses data obtained from patients' medical records as well as a telephone follow-up survey or a review of medical records of patients obtained between 6 and 12 months after discharge. All patients aged 18 years and older admitted with the documented diagnosis of unstable angina or acute myocardial infarction between January 1999 and December 2007 were eligible for the registry. Eligibility criteria for the registry included electrocardiogram changes, elevated cardiac enzymes, and patient-reported symptoms consistent with cardiac ischemia within $24 \mathrm{~h}$ of presentation to the hospital. Data in the registry are abstracted from the index hospitalization by cardiology fellows and entered into the registry by research assistants. Further data on follow-up events are obtained by telephone interview or review of medical records conducted by research assistants. Mortality status at follow-up was validated on all patients through review of the Social Security Death Registry. The registry is approved by the human investigations committee of the affiliated medical school, as is the present study utilizing registry patients.

\section{Dependent variables}

The study end points were death during hospitalization, death during the follow-up period between discharge and telephone follow-up, and occurrence of secondary cardiovascular events or cardiovascular-related procedures during the follow-up period. Cause of death was not specified. Occurrence of a secondary cardiovascular event or cardiovascular-related medical procedure was determined by patient self-report from the follow-up telephone survey. Included in secondary events or procedures were hospitalization for cardiac event; scheduled or unscheduled revascularization procedures such as coronary artery bypass graft or percutaneous coronary intervention; and procedures such as stress tests.

\section{GRACE Acute Coronary Syndrome Risk Prediction Index}

The GRACE index score was calculated for each patient in the ACS registry. The GRACE project was a multinational registry that contains patients who were hospitalized with ACS. $^{18}$

The GRACE database was used to create a risk prediction model that could be used for ACS patients. This risk model is able to predict all-cause mortality in ACS patients. As explained earlier, this model has been used in studies to predict death and/or myocardial infarction at 6 months and longterm mortality after the presentation of ACS. ${ }^{10,14}$ Full details of the GRPI have been described in other reviews. ${ }^{12,18}$ The prediction model was created using Cox proportional hazards regression, which included variables such as patient baseline characteristics, signs and symptoms at presentation, and inhospital treatments, procedures, and complications. ${ }^{12}$ A prediction tool was developed from this data that evaluates the predictor variables from the model and assigns a 
point total based on those variables for each patient. This can then be applied to the reference plot to show the corresponding risk of death using hazard ratios.

\section{Charlson Comorbidity Index}

Charlson's method consists of a weighted index based on the presence of comorbid conditions that predict 1-year survival. ${ }^{9}$ The index was derived from a training population of 559 medical inpatients at New York hospital and validated on a testing population of 685 breast cancer patients at another hospital. As a result, a list of 19 conditions, some representing 2 degrees of severity of the same condition, was designed. Weights were assigned based on relative risk of death, ranging from 1 to 6 . The total score was the sum of the weights, and can range from 0 to 33 . Depending on the version used, there is a list of 17 to 19 conditions. The study demonstrated a strong association with an increase in the 10 -year risk of death in a cohort of breast cancer patients ${ }^{9}$ and similar postoperative survival in patients with hypertension or diabetes. ${ }^{19}$ A version of the CCI was developed by Deyo et al and tested in Medicare beneficiaries who underwent lumbar spine surgery. It was significantly associated with hospital complications. ${ }^{20}$

The version of the CCI used for the present study is known as the D'Hoore version. This version was developed using the International Classification of Diseases, Ninth Revision (ICD-9) codes without the Clinical Modification (CM) codes. $^{21}$ The major differences between the original $\mathrm{CI}$ and the $\mathrm{D}^{\prime}$ Hoore version is the use of only the first 3 digits of the ICD-9 code. Three-digit ICD-9 coding is common in administrative claims data to aggregate data within conditions. For example, no distinction is made between uncomplicated diabetes (ICD-9 250.0) and diabetes with complications (ICD-9 codes 250.1 through 250.9). (The $\mathrm{D}^{\prime}$ Hoore version of the Charlson comorbidity diseases and ICD-9 codes is available from the authors upon request.)

\section{Additional patient characteristics}

Additional information about each patient was abstracted from the ACS registry (ie, age, sex, race). The study was approved by the University of Michigan Medical Institutional Review Board, and patients in the study gave informed consent when enrolled in the registry.

\section{Statistical analysis}

Description of the data is provided using the mean and standard deviation for continuous variables and frequency with percentage for categorical data. GRPI and CCI scores were compared for each of the 3 dependent variables: death inpatient versus alive at discharge; death in follow-up period versus alive in follow-up period; and secondary event during follow-up period versus no event.

The first objective of this study was to compare the predictive ability of the GRPI to that of the CCI on the outcomes of inhospital and follow-up mortality, as well as the occurrence of secondary events or procedures. Logistic regression models were run for the dichotomized outcomes (alive vs. expired in hospital; alive vs. expired in the follow-up period; and secondary event vs. no secondary event) for each of the indexes along with a combined index model. Model parameters used to describe model fit (Hosmer-Lemeshow [H-L] chi-square test), -2 log likelihood were derived for each model. The H-L goodness-of-fit test was used to assess calibration, where the higher the $P$ value, the better the calibration. Model discrimination was assessed by the $\mathrm{C}$ statistic that is equivalent to the area under the receiver operating characteristic curve (ROC). A model with a $\mathrm{C}$ statistic of $>0.75$ is considered to have meaningful discriminatory ability.

The second objective of the study was to determine if adding the CCI to the model containing the GRPI led to improved prediction of the study end point. This was accomplished using the likelihood ratio test. This procedure determines whether the addition of 1 index to a model containing the other index improves the predictive ability over the model that uses 1 index alone. The likelihood ratio test is derived by calculating the difference between the -2 log likelihood values for models that contain single indices and a model that contains both indices (nested model). For example, the difference between the -2 log likelihood values for the models of CCI alone and the CCI plus GRPI model (nested model) is first calculated, then the $P$ value is determined for the chi-square using 1 degree of freedom. If the $P$ value is $<0.05$, the interpretation is that the addition of the GRPI leads to a significant improvement in the predictive ability of the model. This procedure was conducted for each combination of indices and outcomes. SPSS version 18 (IBM, Armonk, NY) and R software were used for analyses.

\section{Results}

The initial group of registry subjects included 1202 patients with complete data from the index hospitalization, including a GRPI. These individuals also were able to have CCI scores calculated. Characteristics of the entire study group are listed in Table 1.

Table 2 provides the comparison of the mean index scores based on the dichotomized dependent variables. In all comparisons, registry patients who either died during the inpatient or follow-up period, or who experienced a

Table 1. Characteristics of the Acute Coronary Syndrome Registry Patients $(N=1202)$

\begin{tabular}{lc}
\hline Variable & $\begin{array}{c}\text { Mean (standard deviation) } \\
\text { or Frequency (percent) Range }\end{array}$ \\
\hline Age-Years & $64.2(13.7)$ \\
Sex-Male & 18.6 to 96.5 \\
Race-White & $781(65.0)$ \\
GRACE Score & $1002(83.4)$ \\
& $105.7(33.8)$ \\
Charlson Co-Morbidity & 20 to 211 \\
Score & $2.3(1.9)$ \\
Death in hospital & 0 to 11 \\
$\quad$ during index event & $55(4.6)$ \\
Death during follow-up & $88(7.7)$ \\
period & \\
Secondary cardiovascular & \\
event or related & \\
procedure during & \\
follow-up period &
\end{tabular}

GRACE, global registry of acute coronary events. 
Table 2. Comparison of Mean Charlson Comorbidity Index Score and GRACE Score

\begin{tabular}{|c|c|c|c|c|}
\hline & & Mean & Score (SD), $n$ & \\
\hline Index & Variable & Alive or no secondary event & Death or experienced a secondary event & Sig. P value \\
\hline \multirow[t]{3}{*}{ Charlson } & $\begin{array}{l}\text { Mortality status as } \\
\text { inpatient }\end{array}$ & $\begin{array}{c}2.23(1.90) \\
n=1147\end{array}$ & $\begin{array}{c}3.45(1.98) \\
\quad n=55\end{array}$ & $<0.001$ \\
\hline & $\begin{array}{l}\text { Mortality status during } \\
\text { post-hospitalization } \\
\text { period }\end{array}$ & $\begin{array}{c}2.13(1.76) \\
n=1059\end{array}$ & $\begin{array}{c}4.23(2.41) \\
\quad n=88\end{array}$ & $<0.001$ \\
\hline & $\begin{array}{l}\text { Secondary event status } \\
\text { during post- } \\
\text { hospitalization period }\end{array}$ & $\begin{array}{c}1.97(1.67) \\
n=503\end{array}$ & $\begin{array}{c}2.61(2.03) \\
n=317\end{array}$ & $<0.001$ \\
\hline \multirow[t]{3}{*}{ GRACE } & $\begin{array}{l}\text { Mortality status as } \\
\text { inpatient }\end{array}$ & $\begin{array}{c}104.45(33.64) \\
n=1147\end{array}$ & $\begin{array}{c}131.33(27.5) \\
n=55\end{array}$ & $<0.001$ \\
\hline & $\begin{array}{l}\text { Mortality status during } \\
\text { post-hospitalization } \\
\text { period }\end{array}$ & $\begin{array}{c}102.25(32.96) \\
n=1059\end{array}$ & $\begin{array}{c}130.85(30.6) \\
n=88\end{array}$ & $<0.001$ \\
\hline & $\begin{array}{l}\text { Secondary event status } \\
\text { during post- } \\
\text { hospitalization period }\end{array}$ & $\begin{array}{c}100.33(32.68) \\
n=503\end{array}$ & $\begin{array}{c}108.27(32.63) \\
n=317\end{array}$ & $<0.001$ \\
\hline
\end{tabular}

${ }^{*} n=$ number of participants experiencing an outcome.

GRACE, Global Registry of Acute Coronary Events.

secondary event or procedure, had higher CCI scores indicating greater comorbidity, as well as higher GRPI scores, indicating greater risk of mortality related to the ACS event.

Multivariate logistic regression modeling results are shown in Table 3, grouped based on the outcome assessed. Table 4 lists the chi-square and $P$ values for the log likelihood ratio tests used to assess the influence of adding the CCI to the GRPI models for each outcome. For death while an inpatient for the index ACS event, the GRPI index model demonstrated the best model fit. Combining the GRPI with the CCI model had the highest value of the 3 models. The $C$ statistic for the GRPI model was highest, indicating greater discriminatory ability of the GRPI over the CCI. The $-2 \log$ likelihood ratio $P$ value was significant when the GRPI was added to the CCI model, but not significant when the CCI was added to the GRPI model. This is interpreted as the CCI adds only slight improvement in predictive ability to the model that already includes the GRPI.

The next outcome, death during the follow-up period, also is described by model statistics in Table 3. The H-L chisquare statistic was greatest for the GRPI regression model, compared to both the CCI alone or in combination with the

Table 3. Logistic Regression Models

\begin{tabular}{|c|c|c|c|c|}
\hline Model & -2 Log Likelihood & H-L Chi-Square, P value & Odds Ratio $(95 \%$ CI) & C statistic $(95 \% \mathrm{CI})$ \\
\hline Inpatient death CCI & 430.63 & $8.16,0.09$ & $1.28(1.14-1.43)$ & 0.68 (0.61 to 0.75$)$ \\
\hline Inpatient death GRACE & 413.21 & $10.41,0.24$ & $1.025(1.02-1.03)$ & $0.73(0.67$ to 0.79$)$ \\
\hline $\begin{array}{l}\text { Inpatient death } \\
\text { combined CCI and } \\
\text { GRACE }\end{array}$ & 408.61 & $8.20,0.41$ & $\begin{array}{l}1.02(1.01-1.03) \\
1.16(1.02-1.31)\end{array}$ & $\begin{array}{c}0.75 \\
p<0.001\end{array}$ \\
\hline Follow-up death CCI & 542.48 & $12.72,0.013$ & $1.55(1.41-1.72)$ & $0.77(0.72-0.82)$ \\
\hline $\begin{array}{l}\text { Follow-up death } \\
\text { GRACE }\end{array}$ & 561.41 & $7.89,0.44$ & $1.03(1.02-1.03)$ & $0.74(0.69-0.79)$ \\
\hline $\begin{array}{l}\text { Follow-up death } \\
\text { combined CCI and } \\
\text { GRACE }\end{array}$ & 515.47 & $17.4,0.026$ & $\begin{array}{l}1.44(1.3-1.6) \\
1.02(1.01-1.03)\end{array}$ & $\begin{array}{c}0.81 \\
p<0.001\end{array}$ \\
\hline $\begin{array}{l}\text { Postdischarge cardiac } \\
\text { event or procedure } \\
\text { CCI }\end{array}$ & 1070.51 & $10.46,0.03$ & $1.21(1.12-1.31)$ & $0.60(0.56-0.64)$ \\
\hline $\begin{array}{l}\text { Postdischarge cardiac } \\
\text { event or procedure } \\
\text { GRACE }\end{array}$ & 1082.82 & $6.82,0.56$ & $1.01(1.003-1.01)$ & $0.57(0.53-0.61)$ \\
\hline $\begin{array}{l}\text { Postdischarge cardiac } \\
\text { event or procedure } \\
\text { combined CCI and } \\
\text { GRACE }\end{array}$ & 1066.79 & $4.66,0.79$ & $\begin{array}{c}1.09(1.02-1.16) \\
1.006(1.002-1.01)\end{array}$ & $\begin{array}{c}0.58 \\
p<0.001\end{array}$ \\
\hline
\end{tabular}

CCI, Charlson Comorbidity Index; GRACE, Global Registry of Acute Coronary Events; H-L, Hosmer-Lemeshow Test. 
Table 4. -2 Log Likelihood Ratio Test

\begin{tabular}{lcc}
\hline Index model description & Chi-square value & P value \\
\hline Inpatient mortality & & \\
CCI and combined & 22.013 & $<0.001$ \\
GRACE and combined & 4.594 & 0.03 \\
Follow-up period mortality & & \\
CCI and combined & 27.009 & $<0.001$ \\
GRACE and combined & 18.934 & $<0.001$ \\
Secondary cardiovascular event or related procedure \\
CCI and combined & 3.726 & 0.05 \\
GRACE and combined & 16.031 & $<0.001$ \\
\hline
\end{tabular}

CCI, Charlson Comorbidity Index; GRACE, Global Registry of Acute Coronary Events Index.

GRPI. The C statistic for the CCI was highest for the CCI model, followed closely by the GRPI model $C$ statistic. This indicates that both indices have similar ability to discriminate the outcome of death during the follow-up period. The $-2 \log$ likelihood ratio test demonstrated that there was significant improvement to each model when combined with the other index.

The models for the outcome of experiencing a secondary event or procedure during the follow-up period demonstrated that the GRPI alone model as well as the combined GRPI with CCI had relatively high $P$ values for the $\mathrm{H}-\mathrm{L}$ chisquare test, indicating acceptable model calibration. The C statistic for the CCI model was marginally greater than the GRPI, both being below that important value of 0.7 . The -2 log likelihood ratio test indicated that adding the CCI to a model containing the GRPI significantly improved model predictive ability, while adding the GRPI to a model starting with the CCI alone only marginally improved model performance.

\section{Discussion}

Risk indices such as the GRPI are developed to assist the clinician when determining the type and intensity of current and near-term clinical interventions that may be necessary for patients who have experienced a medical event. Riskadjustment indices also are used by health care administrators to allocate resources based on patient acuity.

The GRPI needs no assistance to predict mortality of patients with ACS during the index hospitalization. The GRPI, with a $\mathrm{C}$ statistic above 0.7 , was greater than the CCI's C statistic, which was less than 0.7, while the log likelihood ratio analysis demonstrated that adding the CCI to a model with GRPI already included did not greatly improve its predictive ability. The GRPI model was originally developed to predict mortality during the inpatient stay, and is useful as a risk stratification tool for clinicians to use to determine therapies. Other work examining the GRPI has demonstrated similar or higher $\mathrm{C}$ statistics. $^{22}$

GRPI and CCI offer similar predictions of outcomes after discharge. The $\mathrm{C}$ statistic for each index model was similar for mortality after discharge as well as for occurrence of secondary events or procedures. In fact, the CCI had a greater association with each outcome. The log likelihood ratio analysis demonstrated that adding the CCI to models containing GRPI significantly improved the predictive ability over the GRPI alone model, while adding GRPI to models that initially included only the CCI did not improve predictive performance. Over the 6-month follow-up period, the CCI was better at predicting both death and a secondary cardiovascular event in comparison to GRPI. This may be a function of the important role that comorbidity plays in determining patient outcomes, and the fact that comorbidities are not mutually exclusive in their association with longer term outcomes. This is not surprising given that the CCI has been well established as an index with excellent long-term predictive value. Charlson et al demonstrated that the model was able to predict mortality in breast cancer patients for up to 10 years. Furthermore, patients with a higher ranking comorbidity exhibited a higher risk of mortality at follow-up. ${ }^{9}$

The general findings of this study are that the GRPI is adequate to predict mortality for the inpatient index event as well as during a period of 6 to 10 months after discharge. It is, by itself, an effective risk adjustment for predicting secondary events after discharge. Adding the CCI to models using the GRPI is useful to improve the prediction of outpatient mortality or secondary events after discharge. The CCI proved to be equally useful in predicting these outcomes if used alone.

The important point is that the clinical data necessary to derive an index score such as the GRPI are not always available to the health services researcher, the population health manager, or the clinician. The data necessary to calculate the GRPI require access to the medical record for manual extraction or extensive programming to extract from a health system's data warehouse. Often, however, data are available on diagnoses for individual patients, particularly in administrative claims data or clinical summary documents in the medical record. Using the CCI, or a similar diagnosisderived index, may be useful in situations in which the clinical data comprising the GRPI model are not available.

This study has several limitations. A retrospective analysis of existing registry data such as this includes problems of variability in physician documentation; specifically, omission of diagnoses in the emergency and hospital setting that may have led to lower than expected correlations between predicted and observed outcomes. Another limitation is that the contribution of specific diseases within the CCI to the outcomes was not studied. There may be a subset of conditions within the CCI that have the greatest association with the outcomes. In addition, using a predefined set of conditions limits the ability to detect the association of non-included diagnoses on the outcome of interest. There is a limitation when using a single-value index to control for the influence of comorbid conditions, because it does not allow the researcher to directly assess the individual influence of specific diseases that make up the index. However, if the purpose of including a single-value index is strictly to control for potential variation related to these conditions, allowing the analyst to focus on other, hypothesized important variables, then use of the CCI plus GRPI is appropriate.

\section{Conclusion}

The findings presented here suggest that the CCI would be a useful addition to the GRPI when analyzing future cardiac-related events or mortality after discharge for an ACS event. 


\section{Author Disclosure Statement}

Drs. Erickson, Cole, and Eagle, and Ms. Kline-Rogers declared no conflicts of interest, nor financial support, with respect to the research, authorship, and/or publication of this article.

\section{References}

1. Go AS, Mozaffarian D, Roger VL, et al. American Heart Association Statistics Committee and Stroke Statistics Subcommittee. Heart disease and stroke statistics—2013 update: A report from the American Heart Association. Circulation 2013;127:e6-e245.

2. Taneva E, Bogdanova V, Shtereva N. Acute coronary syndrome, co-morbidity, and mortality in geriatric patients. Ann NY Acad Sci 2004;1019:106-110.

3. Menotti A, Mulder I, Nissinen A, Giampaoli S, Feskens EJ, Kromhout D. Prevalence of morbidity and multimorbidity in elderly male populations and their impact on 10-year allcause mortality: The FINE study (Finland, Italy, Netherlands, Elderly). J Clin Epidemiol 2001;54:680-686.

4. Lee T, Shields A, Vogeli C, et al. Mortality rate in veterans with multiple chronic conditions. J Gen Intern Med 2007;22:403-407.

5. Glynn LG, Buckley B, Reddan D, et al. Multimorbidity and risk among patients with established cardiovascular disease: A cohort study. Br J Gen Pract 2008;58:488-494.

6. Lichtman JH, Fathi A, Radford MJ, Lin Z, Loeser CS, Krumholz HM. Acute, severe noncardiac conditions in patients with acute myocardial infarction. Am J Med 2006;119:843-850.

7. Stukenborg GJ, Wagner DP, Harrell FE, et al. Present-atadmission diagnoses improved mortality risk adjustment among acute myocardial infarction patients. J Clin Epidemiol 2007;60:142-154.

8. Sachdev M, Sun JL, Tsiatis AA, Nelson CL, Mark DB, Jollis JG. The prognostic importance of co-morbidity for mortality in patients with stable coronary artery disease. J Am Coll Cardiol 2004;43:576-582.

9. Charlson ME, Pompei P, Ales KL, MacKenzie CR. A new method of classifying prognostic co-morbidity in longitudinal studies: Development and validation. J Chronic Dis 1987;40:373-383.

10. Tang EW, Wong CK, Herbison P. Global Registry of Acute Coronary Events (GRACE) hospital discharge risk score accurately predicts long-term mortality post acute coronary syndrome. Am Heart J 2007;153:29-35.

11. Granger CB, Goldberg RJ, Dabbous O, et al. Predictors of hospital mortality in the Global Registry of Acute Coronary Events. Arch Intern Med. 2003;163:2345-2353.

12. Eagle KA, Lim MJ, Dabbous $\mathrm{OH}$, et al. A validated prediction model for all forms of acute coronary syndrome. Esti- mating the risk of 6-month postdischarge death in an international registry. JAMA 2004;291:2727-2733.

13. Yan AT, Yan RT, Tan M, et al. In-hospital revascularization and 1-year outcome of acute coronary syndrome patients stratified by the GRACE risk score. Am J Cardiol 2005;96:913-916.

14. Fox KAA, Dabbous OH, Goldberg RJ, et al. Prediction of risk of death and myocardial infarction in the six months after presentation with acute coronary syndrome: Prospective multinational observational study (GRACE). BMJ 2006;333: 1091-1094.

15. Beygui F, Silvain J, Pena A, et al. Usefulness of biomarker strategy to improve GRACE score's prediction performance in patients with non-ST-segment elevation acute coronary syndrome and low event rates. Am J Cardiol 2010;106:650 658.

16. Ang DSC, Wei L, Kao MPC, Lang CC, Struther AD. A comparison between Bb-type natuiuretic peptide, Global Registry of Acute Coronary Events (GRACE) score and their combination in ACS risk stratification. Heart 2009;95:18361842.

17. Eggers KM, Kempf T, Venge P, Wallentin L, Wollert KC, Lindahl B. Improving long-term risk prediction in patients with acute chest pain: The Global Registry of Acute Coronary Events (GRACE) risk score is enhanced by selected nonnecrosis biomarkers. Am Heart J 2010;160:88-94.

18. GRACE Investigators. Rationale and design of the GRACE (global registry of acute coronary events) project: A multinational registry of patients hospitalized with acute coronary syndrome. Am Heart J 2001;141:190-199.

19. Charlson ME, Szatrowski TP, Peterson J, Gold J. Validation of a combined co-morbiditty index. J Clin Epidemiol 1994;47:1245-1251.

20. Deyo RA, Cherkin DC, Ciol MA. Adapting a clinical comorbidity index for use with ICD-9-CM administrative databases. J Clin Epidemiol 1992;45:613-619.

21. D'Hoore W, Bouckaert A, Tilquin C. Practical considerations on the use of the Charlson index with administrative data bases. J Clin Epidemiol 1996;49:1429-1433.

22. Pieper KS, Gore JM, FitzGerald G, et al. Global Registry of Acute Coronary Events (GRACE) Investigators. Validity of a risk-prediction tool for hospital mortality: The Global Registry of Acute Coronary Events. Am Heart J 2009;157:1097-1105.

Address correspondence to:

Steven R. Erickson, PharmD University of Michigan College of Pharmacy 428 Church Street Ann Arbor, MI 48109-1065

E-mail: serick@umich.edu 


\section{This article has been cited by:}

1. Muhammad Rashid, Chun Shing Kwok, Chris P. Gale, Patrick Doherty, Ivan Olier, Matthew Sperrin, Evangelos Kontopantelis, George Peat, Mamas A. Mamas. 2017. Impact of co-morbid burden on mortality in patients with coronary heart disease, heart failure, and cerebrovascular accident: a systematic review and meta-analysis. European Heart Journal - Quality of Care and Clinical Outcomes 3:1, 20-36. [CrossRef]

2. Paolo Fraccaro, Evangelos Kontopantelis, Matthew Sperrin, Niels Peek, Christian Mallen, Philip Urban, Iain E. Buchan, Mamas A. Mamas. 2016. Predicting mortality from change-over-time in the Charlson Comorbidity Index. Medicine 95:43, e4973. [CrossRef]

3. Yaser M.K. Baghdadi, David S. Morris, Asad J. Choudhry, Cornelius A. Thiels, Mohammad A. Khasawneh, Stephanie F. Polites, Donald H. Jenkins, Martin D. Zielinski. 2016. Validation of the anatomic severity score developed by the American Association for the Surgery of Trauma in small bowel obstruction. Journal of Surgical Research . [CrossRef]

4. Joakim Alfredsson, Karen P. Alexander. 2016. Multiple Chronic Conditions in Older Adults with Acute Coronary Syndromes. Clinics in Geriatric Medicine 32:2, 291-303. [CrossRef]

5. Linda Worrall-Carter, Samantha McEvedy, Andrew Wilson, Muhammad Aziz Rahman. 2016. Impact of comorbidities and gender on the use of coronary interventions in patients with high-risk non-ST-segment elevation acute coronary syndrome. Catheterization and Cardiovascular Interventions 87:4, E128-E136. [CrossRef]

6. Mamas A. Mamas, Farzin Fath-Ordoubadi, Gian B. Danzi, Erik Spaepen, Chun Shing Kwok, Iain Buchan, Niels Peek, Mark A. de Belder, Peter F. Ludman, Dragica Paunovic, Philip Urban. 2015. Prevalence and Impact of Co-morbidity Burden as Defined by the Charlson Co-morbidity Index on 30-Day and 1- and 5-Year Outcomes After Coronary Stent Implantation (from the Nobori-2 Study). The American Journal of Cardiology 116:3, 364-371. [CrossRef]

7. Matthias Michal, Jürgen H. Prochaska, Karsten Keller, Sebastian Göbel, Meike Coldewey, Alexander Ullmann, Andreas Schulz, Heidrun Lamparter, Thomas Münzel, Iris Reiner, Manfred E. Beutel, Philipp S. Wild. 2015. Symptoms of depression and anxiety predict mortality in patients undergoing oral anticoagulation: Results from the thrombEVAL study program. International Journal of Cardiology 187, 614-619. [CrossRef] 